\title{
A Fresh Approach to Learning Register Automata ${ }^{\star}$
}

\author{
Benedikt Bollig ${ }^{1}$, Peter Habermehl ${ }^{2}$, Martin Leucker ${ }^{3}$, and Benjamin \\ Monmege $^{1}$ \\ 1 LSV, ENS Cachan, CNRS \& Inria, France \\ 2 Univ Paris Diderot, Sorbonne Paris Cité, LIAFA, CNRS, France \\ ${ }^{3}$ ISP, University of Lübeck, Germany
}

\begin{abstract}
This paper provides an Angluin-style learning algorithm for a class of register automata supporting the notion of fresh data values. More specifically, we introduce session automata which are well suited for modeling protocols in which sessions using fresh values are of major interest, like in security protocols or ad-hoc networks. We show that session automata (i) have an expressiveness partly extending, partly reducing that of register automata, (ii) admit a symbolic regular representation, and (iii) have a decidable equivalence and model-checking problem (unlike register automata). Using these results, we establish a learning algorithm to infer session automata through membership and equivalence queries. Finally, we strengthen the robustness of our automaton by its characterization in monadic second-order logic.
\end{abstract}

\section{Introduction}

Learning automata deals with the inference of automata based on some partial information, for example samples, which are words that either belong to their accepted language or not. A popular framework is that of active learning defined by Angluin [2] in which a learner may consult a teacher for so-called membership and equivalence queries to eventually infer the automaton in question. Learning automata has a lot of applications in computer science. Notable examples are the use in model checking [12] and testing [3]. See [18] for an overview.

While active learning of regular languages is meanwhile well understood and is supported by freely available libraries such as learnlib [19] and libalf [8], extensions beyond plain regular languages are still an area of active research. Recently, automata dealing with potentially infinite data as first class citizens have been studied. Seminal works in this area are that of $[1,15]$ and [14]. While the first two use abstraction and refinement techniques to cope with infinite data, the second approach learns a sub-class of register automata.

In this paper, we follow the work on learning register automata. However, we study a different model than [14], having the ability to require that input data is fresh in the sense that it has not been seen so far. This feature has been proposed in [24] in the context of semantics of programming languages, as, for

\footnotetext{
^ This work is partially supported by EGIDE/DAAD-Procope (LeMon).
} 
example, fresh names are needed to model object creation in object-oriented languages. Moreover, fresh data values are important ingredients in modeling security protocols which often make use of so-called fresh nonces to achieve their security assertions [17]. Finally, fresh names are also important in the field of network protocols and are one of the key ingredients of the $\pi$-calculus [20].

In general, the equivalence problem of register automata is undecidable (even without freshness). This limits their applicability in active learning, as equivalence queries cannot be implemented (correctly and completely). Therefore, we restrict the studied automaton model to either store fresh data values or read data values from registers. In the terminology of [24], we retain global freshness, while local freshness is discarded. We call our model session automata. They are well-suited whenever fresh values are important for a finite period, for which they will be stored in one of the registers. Session automata correspond to the model from [7] without stacks. They are incomparable with the model from [14].

Session automata accept data words, i.e., words over an alphabet $\Sigma \times D$, where $\Sigma$ is a finite set of labels and $D$ an infinite set of data values. A data word can be mapped to a so-called symbolic word where we record for each different data value the register in which it was stored (when appearing for the first time) or from which it was read later. To each symbolic word we define a symbolic word in unique normal form representing the same data words by fixing a canonical way of storing data values in registers. Then, we show how to transform a session automaton into a unique canonical automaton that accepts the same data language. This canonical automaton can be seen as a classical finite-state automaton and, therefore, we can define an active learning algorithm for session automata in a natural way. In terms of the size of the canonical automaton, the number of membership and equivalence queries needed is polynomial (both in the number of states and in the number of registers). When the reference model are arbitrary (data) deterministic automata, the complexity is polynomial in the number of states and exponential in the number of registers.

Applicability of our framework in verification (e.g., compositional verification [10] and infinite state regular model checking [13]) is underpinned by the fact that session automata form a robust language class: While inclusion is undecidable for register automata [21], we show that it is decidable for session automata. In [7], model checking session automata was shown decidable wrt. a powerful monadic second-order logic with data-equality predicate (dMSO). Here, we also provide a natural fragment of dMSO that precisely captures session automata.

To summarize, we show that session automata (i) have a unique canonical form, (ii) have a decidable inclusion problem, (iii) enjoy a logical characterization, and (iv) can be learned via an active learning algorithm. Altogether, this provides a versatile learning framework for languages over infinite alphabets.

Outline. The paper is structured as follows. In Section 2 we introduce session automata. In Section 3 we present an active learning algorithm for them and in Section 4 we give some language-theoretic properties of our model and a logical characterization. Missing proofs can be found in the appendix. 


\section{Data Words and Session Automata}

We let $\mathbb{N}$ (respectively, $\mathbb{N}_{>0}$ ) be the set of natural numbers (respectively, nonzero natural numbers). For $n \in \mathbb{N}$, we let $[n]$ denote the set $\{1, \ldots, n\}$. In the following, we fix a non-empty finite alphabet $\Sigma$ of labels and an infinite set $D$ of data values. In examples, we usually use $D=\mathbb{N}$. A data word is a sequence of elements of $\Sigma \times D$, i.e., an element from $(\Sigma \times D)^{*}$. An example data word over $\Sigma=\{a, b\}$ and $D=\mathbb{N}$ is $(a, 4)(b, 2)(b, 4)$.

Our automata will not be able to distinguish between data words that are equivalent up to permutation of data values. Intuitively, this corresponds to saying that data values can only be compared wrt. equality. When two data words $w_{1}$ and $w_{2}$ are equivalent in that sense, we write $w_{1} \approx w_{2}$, e.g. $(a, 4)(b, 2)(b, 4) \approx$ $(a, 2)(b, 5)(b, 2)$. The equivalence class of a data word $w$ wrt. $\approx$ is written $[w]_{\approx}$.

We can view a data word as being composed of (not necessarily disjoint) sessions, each session determining the scope in which a given data value is used. Let $w=\left(a_{1}, d_{1}\right) \cdots\left(a_{n}, d_{n}\right) \in(\Sigma \times D)^{*}$ be a data word. We let Fresh $(w) \stackrel{\text { def }}{=}\{i \in$ $[n] \mid d_{i} \neq d_{j}$ for all $\left.j \in\{1, \ldots, i-1\}\right\}$ be the set of positions of $w$ where a data value occurs for the first time. Accordingly, we let $\operatorname{Last}(w) \stackrel{\text { def }}{=}\left\{i \in[n] \mid d_{i} \neq d_{j}\right.$ for all $j \in\{i+1, \ldots, n\}\}$. A set $S \subseteq[n]$ is a session of $w$ if there are $i \in \operatorname{Fresh}(w)$ and $j \in \operatorname{Last}(w)$ such that $S=\{i, \ldots, j\}$ and $d_{i}=d_{j}$. For $i \in[n]$, let $\operatorname{Session}(i)$ denote the unique session $S$ with $d_{\min (S)}=d_{i}$. Thus $\operatorname{Session}(i)$ is the scope in which $d_{i}$ is used. Note that $\operatorname{Fresh}(w)=\{\min (\operatorname{Session}(i)) \mid i \in[n]\}$. For $k \geq 1$, we say that $w$ is $k$-bounded if every position of $w$ belongs to at most $k$ sessions. A language $L$ is $k$-bounded if every word in $L$ is so. The set of all data words is not $k$-bounded, for any $k$. Fig. 1 illustrates a data word $w$ with four sessions. It is 2-bounded, as no position shares more than 2 sessions. We have $\operatorname{Session}(7)=\{4, \ldots, 9\}$ and $\operatorname{Fresh}(w)=\{1,2,4,6\}$.

Intuitively, $k$ is the number of resources that will be needed to execute a $k$-bounded word. Speaking in terms of automata, a resource is a register that can store a data value. Our automata will be able to write a fresh data value into some register $r$, denoted $\mathrm{f}(r)$, or reuse a data value that has already been stored in $r$, denoted $r(r)$. In other words, automata will work over (a finite subset of) the alphabet $\Sigma \times \Gamma$ where $\Gamma \stackrel{\text { def }}{=}\left\{\mathrm{f}(r), \mathrm{r}(r) \mid r \in \mathbb{N}_{>0}\right\}$. A word over $\Sigma \times \Gamma$ is called a symbolic word. Given a symbolic word $u=\left(a_{1}, t_{1}\right) \cdots\left(a_{n}, t_{n}\right)$ and a position $i \in[n]$, we let $\operatorname{reg}(i)$ denote the register $r$ that is used at $i$, i.e., such that $t_{i} \in\{\mathrm{f}(r), \mathrm{r}(r)\}$. Similarly, we define the type type $(i) \in\{\mathrm{f}, \mathrm{r}\}$ of $i$.

Naturally, a register has to be initialized before it can be used. So, we call $u$ well formed if, for all $j \in[n]$ with $\operatorname{type}(j)=\mathrm{r}$, there is $i \leq j$ such that

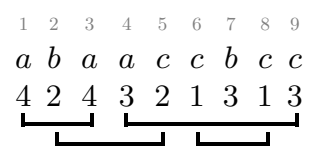

Fig. 1: A data word and its sessions

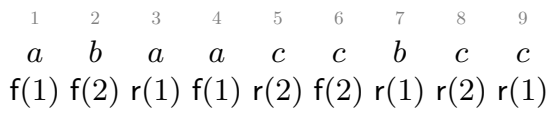

Fig. 2: A symbolic word 
$t_{i}=\mathrm{f}(\operatorname{reg}(j))$. Let WF denote the set of well formed words. A well formed symbolic word is illustrated in Fig. 2. We have type $(5)=\mathrm{r}$ and $\operatorname{reg}(5)=2$.

A symbolic word $u=\left(a_{1}, t_{1}\right) \cdots\left(a_{n}, t_{n}\right) \in \mathrm{WF}$ generates a set of data words. Intuitively, a position $i$ with $t_{i}=\mathrm{f}(r)$ opens a new session, writing a fresh data value in register $r$. The same data value is reused at positions $j>i$ with $t_{j}=\mathrm{r}(r)$, unless $r$ is reinitialized at some position $i^{\prime}$ with $i<i^{\prime}<j$. Formally, $w \in(\Sigma \times D)^{*}$ is a concretization of $u$ if it is of the form $\left(a_{1}, d_{1}\right) \cdots\left(a_{n}, d_{n}\right)$ such that, for all $i, j \in[n]$ with $i \leq j$, (i) $i \in \operatorname{Fresh}(w)$ iff type $(i)=\mathrm{f}$, and (ii) $d_{i}=d_{j}$ iff both $\operatorname{reg}(i)=\operatorname{reg}(j)$ and there is no position $i^{\prime}$ with $i<i^{\prime} \leq j$ such that $t_{i^{\prime}}=\mathrm{f}(\operatorname{reg}(i))$. For example, the data word from Fig. 1 is a concretization of the symbolic word from Fig. 2. By $\gamma(u)$, we denote the set of concretizations of a well formed word $u$. We extend $\gamma$ to sets $L \subseteq(\Sigma \times \Gamma)^{*}$ and let $\gamma(L) \stackrel{\text { def }}{=}\{\gamma(u) \mid u \in L \cap \mathrm{WF}\}$.

Remark 1. Let us state some simple properties of $\gamma$. It is easily seen that $w \in$ $\gamma(u)$ implies $\gamma(u)=[w] \approx$. Let $k \geq 1$. If $u \in \mathrm{WF} \cap\left(\Sigma \times \Gamma_{k}\right)^{*}$ where $\Gamma_{k} \stackrel{\text { def }}{=}$ $\{\mathrm{f}(r), \mathrm{r}(r) \mid r \in[k]\}$, then all data words in $\gamma(u)$ are $k$-bounded. Moreover, $\gamma\left(\left(\Sigma \times \Gamma_{k}\right)^{*}\right)$ is the set of all $k$-bounded data words.

Session Automata. As suggested, we consider automata over the alphabet $\Sigma \times \Gamma$ to process data words. Actually, they are equipped with a finite number $k \geq 1$ of registers so that we rather deal with finite automata over $\Sigma \times \Gamma_{k}$.

Definition 1. Let $k \geq 1$. A $k$-register session automaton (or just session automaton) over $\Sigma$ and $D$ is a finite automaton over $\Sigma \times \Gamma_{k}$, i.e., a tuple $\mathcal{A}=$ $\left(Q, q_{0}, F, \delta\right)$ where $Q$ is the finite set of states, $q_{0} \in Q$ the initial state, $F \subseteq Q$ the set of accepting states, and $\delta: Q \times\left(\Sigma \times \Gamma_{k}\right) \rightarrow 2^{Q}$ the transition function.

The symbolic language $L_{\text {symb }}(\mathcal{A}) \subseteq\left(\Sigma \times \Gamma_{k}\right)^{*}$ of $\mathcal{A}$ is defined in the usual way, considering $\mathcal{A}$ as a finite automaton. Its (data) language is $L_{\text {data }}(\mathcal{A}) \stackrel{\text { def }}{=}$ $\gamma\left(L_{\text {symb }}(\mathcal{A})\right)$. By Remark $1, L_{\text {data }}(\mathcal{A})$ is closed under $\approx$. Moreover, it is $k$ bounded, which motivates the naming of our automata.

Example 1. Consider the 2-register session automaton $\mathcal{A}$ from Fig. 3(a). It recognizes the set of all 2-bounded data words over $\Sigma=\{a\}$.

Example 2. The 2-register session automaton $\mathcal{B}$ over $\Sigma=\{a, b\}$ from Fig. 3(b) represents a client-server system. A server can receive requests on two channels of capacity 1 , represented by the two registers. Requests are acknowledged in the order in which they are received. When the automaton performs $(a, \mathrm{f}(r))$, a client gets a unique transaction key, which is stored in $r$. Later, the request is acknowledged performing $(b, r(r))$. E.g., $(a, 8)(a, 4)(b, 8)(a, 3)(b, 4)(b, 3) \in L_{\text {data }}(\mathcal{B})$.

Example 3. Next, we present a 2-register session automaton that models a P2P protocol. A user can join a host with address $x$, denoted by action $($ join, $x)$. The request is either forwarded by $x$ to another host $y$, executing $\left(\right.$ for $\left._{1}, x\right)\left(\right.$ for $\left._{2}, y\right)$, or acknowledged by $(a c k, x)$. In the latter case, a connection between the user and $x$ is established so that they can communicate, indicated by action $(\operatorname{com}, x)$. 


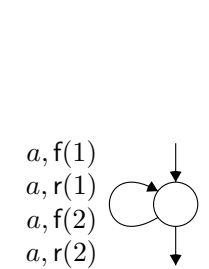

(a) $\mathcal{A}$

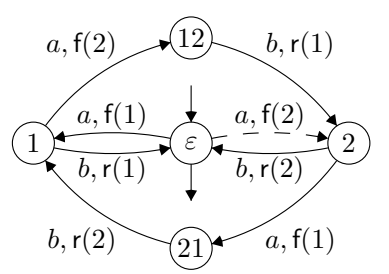

(b) $\mathcal{B}$ and $\mathcal{B}^{\prime}$

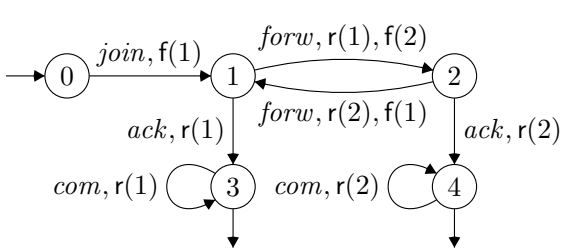

(c)

Fig. 3: (a) Session automaton, (b) Client-server system, (c) P2P protocol

Note that the sequence of actions $\left(\right.$ for $\left._{1}, x\right)\left(\right.$ for $\left._{2}, y\right)$ should be considered as an encoding of a single action (forw, $x, y)$ and is a way of dealing with actions that actually take two or more data values. An example execution of our protocol is $($ join, 145$)($ forw $, 145,978)($ forw $, 978,14)($ ack, 14$)($ com, 14$)($ com, 14$)($ com, 14$)$. In Fig. 3(c), we show the 2-register session automaton for the P2P protocol.

Session automata come with two natural notions of determinism. We call $\mathcal{A}=\left(Q, q_{0}, F, \delta\right)$ symbolically deterministic if $|\delta(q,(a, t))| \leq 1$ for all $q \in Q$ and $(a, t) \in \Sigma \times \Gamma_{k}$. Then, $\delta$ can be seen as a partial function $Q \times\left(\Sigma \times \Gamma_{k}\right) \rightarrow Q$. We call $\mathcal{A}$ data deterministic if it is symbolically deterministic and, for all $q \in Q$, $a \in \Sigma$, and $r_{1}, r_{2} \in[k]$ with $r_{1} \neq r_{2}$, we have that $\delta\left(q,\left(a, \mathrm{f}\left(r_{1}\right)\right)\right) \neq \emptyset$ implies $\delta\left(q,\left(a, \mathrm{f}\left(r_{2}\right)\right)\right)=\emptyset$. Intuitively, given a data word as input, the automaton is data deterministic if, in each state, given a pair letter/data value, there is at most one fireable transition. While "data deterministic" implies "symbolically deterministic", the converse is not true. E.g., the session automata from Fig. 3(a) and $3(\mathrm{~b})$ are symbolically deterministic but not data deterministic. However, the automaton of Fig. 3(b) with the dashed transition from state $\varepsilon$ to state 2 removed is data deterministic.

Theorem 1. Session automata are strictly more expressive than data deterministic session automata.

Session automata are expressively incomparable with the various register automata models considered in $[16,21,23,9,14]$. In particular, due to freshness, the languages from Ex. 1, 2, and 3 are not recognizable by the models for which a learning algorithm exists $[9,14]$. On the other hand, our model cannot recognize "the set of all data words" or "every two consecutive data values are distinct". Our automata are subsumed by fresh-register automata [24], class memory automata [5], and data automata [6]. However, no algorithm for the inference of the latter is known. Note that, for ease of presentation, we consider one-dimensional data words, unlike [14] where labels have an arity and can carry several data values. Following [7], our automata can be easily extended to multi-dimensional data words (cf. Ex. 3). This also holds for the learning algorithm.

Canonical Session Automata. Our goal will be to infer the data language of a session automaton $\mathcal{A}$ in terms of a canonical session automaton $\mathcal{A}^{C}$, which we develop in the following. 
As a first step, we associate with a data word $w=\left(a_{1}, d_{1}\right) \cdots\left(a_{n}, d_{n}\right) \in$ $(\Sigma \times D)^{*}$ a symbolic normal form snf $(w) \in \mathrm{WF}$ such that $w \in \gamma(\operatorname{snf}(w))$, based on the idea that data values are always stored in the first register whose data value is not needed anymore. To do so, we will determine $t_{1}, \ldots, t_{n} \in \Gamma$ and set $\operatorname{snf}(w)=\left(a_{1}, t_{1}\right) \cdots\left(a_{n}, t_{n}\right)$. We define $\tau: \operatorname{Fresh}(w) \rightarrow \mathbb{N}_{>0}$ inductively by $\tau(i)=\min ($ FreeReg $(i))$ where FreeReg $(i) \stackrel{\text { def }}{=} \mathbb{N}_{>0} \backslash\left\{\tau\left(i^{\prime}\right) \mid i^{\prime} \in \operatorname{Fresh}(w)\right.$ such that $i^{\prime}<i$ and $\left.i \in \operatorname{Session}\left(i^{\prime}\right)\right\}$. With this, we set, for all $i \in[n], t_{i}=\mathrm{f}(\tau(i))$ if $i \in \operatorname{Fresh}(w)$, and $t_{i}=\mathrm{r}(\tau(\min (\operatorname{Session}(i))))$ otherwise. One readily verifies that $\operatorname{snf}(w)=\left(a_{1}, t_{1}\right) \cdots\left(a_{n}, t_{n}\right)$ is well formed and that properties (i) and (ii) in the definition of a concretization hold. This proves $w \in \gamma(\operatorname{snf}(w))$. E.g., Fig. 2 shows the symbolic normal form of the data word from Fig. 1. The mapping snf carries over to languages in the expected manner.

We consider again $\mathcal{B}$ of Fig. 3(b). Let $\mathcal{B}^{\prime}$ be the automaton that we obtain from $\mathcal{B}$ when we remove the dashed transition. We have $L_{\text {data }}(\mathcal{B})=L_{\text {data }}\left(\mathcal{B}^{\prime}\right)$, but $\operatorname{snf}\left(L_{\text {data }}(\mathcal{B})\right)=L_{\text {symb }}\left(\mathcal{B}^{\prime}\right) \varsubsetneqq L_{\text {symb }}(\mathcal{B})$.

Lemma 1. Let $L$ be a regular language over $\Sigma \times \Gamma_{k}$. Then, $\operatorname{snf}(\gamma(L))$ is a regular language over $\Sigma \times \Gamma_{k}$.

In other words, for every $k$-register session automaton $\mathcal{A}$, there is a $k$-register session automaton $\mathcal{A}^{\prime}$ such that $L_{\text {symb }}\left(\mathcal{A}^{\prime}\right)=\operatorname{snf}\left(L_{\text {data }}(\mathcal{A})\right)$ and, therefore, $L_{\text {data }}\left(\mathcal{A}^{\prime}\right)=L_{\text {data }}(\mathcal{A})$. We denote by $\mathcal{A}^{C}$ the minimal symbolically deterministic automaton $\mathcal{A}^{\prime}$ satisfying $L_{\text {symb }}\left(\mathcal{A}^{\prime}\right)=\operatorname{snf}\left(L_{\text {data }}(\mathcal{A})\right)$. Note that the number $k^{\prime}$ of registers effectively used in $\mathcal{A}^{C}$ may be smaller than $k$, and we actually consider $\mathcal{A}^{C}$ to be a $k^{\prime}$-register session automaton.

Theorem 2. Let $\mathcal{A}=\left(Q, q_{0}, F, \delta\right)$ be a k-register session automaton. Then, $\mathcal{A}^{C}$ has at most $2^{O\left(|Q| \times(k+1) ! \times 2^{k}\right)}$ states. If $\mathcal{A}$ is data deterministic, then $\mathcal{A}^{C}$ has at most $O\left(|Q| \times(k+1) ! \times 2^{k}\right)$ states. Finally, $\mathcal{A}^{C}$ uses at most $k$ registers.

\section{Learning Session Automata}

In this section, we introduce an active learning algorithm for session automata. In the usual active learning setting (as introduced by Angluin [2]), a learner interacts with a so-called minimally adequate teacher (MAT), an oracle which can answer membership and equivalence queries. In our case, the learner is given the task to infer the data language $L_{\text {data }}(\mathcal{A})$ defined by a given session automaton $\mathcal{A}$. We suppose here that the teacher knows the session automaton or any other device accepting $L_{\text {data }}(\mathcal{A})$. In practice, this might not be the case $-\mathcal{A}$ could be a black box - and equivalence queries could be (approximately) answered, for example, by extensive testing. The learner can ask if a data word is accepted by $\mathcal{A}$ or not. Furthermore it can ask equivalence queries which consist in giving an hypothesis session automaton to the teacher who either answers yes, if the hypothesis is equivalent to $\mathcal{A}$ (i.e., both data languages are the same), or gives a data word which is a counterexample, i.e., a data word that is either accepted by the hypothesis automaton but should not, or vice versa. 
Given the data language $L_{\text {data }}(\mathcal{A})$ accepted by a session automaton $\mathcal{A}$ over $\Sigma$ and $D$, our algorithm will learn the canonical $k$-register session automaton $\mathcal{A}^{C}$, i.e., the minimal symbolically deterministic automaton recognizing the data language $L_{\text {data }}(\mathcal{A})$ and the regular language $L_{\text {symb }}\left(\mathcal{A}^{C}\right)$ over $\Sigma \times \Gamma_{k}$. Therefore one can consider that the learning target is $L_{\text {symb }}\left(\mathcal{A}^{C}\right)$ and use any active learning algorithm for regular languages. However, as the teacher answers only questions over data words, queries have to be adapted. Since $\mathcal{A}^{C}$ only accepts symbolic words which are in normal form, a membership query for a given symbolic word $u$ not in normal form will be answered negatively (without consulting the teacher); otherwise, the teacher will be given one data word included in $\gamma(u)$ (all the answers on words of $\gamma(u)$ are the same). Likewise, before submitting an equivalence query to the teacher, the learning algorithm checks if the current hypothesis automaton accepts symbolic words not in normal form ${ }^{4}$. If yes, one of those is taken as a counterexample, else an equivalence query is submitted to the teacher. Since the number of registers needed to accept a data language is a priori not known, the learning algorithm starts by trying to learn a 1-register session automaton and increases the number of registers as necessary.

Any active learning algorithm for regular languages may be adapted to our setting. Here we describe a variant of Rivest and Schapire's [22] algorithm which is itself a variant of Angluin's L* algorithm [2]. An overview of learning algorithms for deterministic finite state automata can be found, for example, in [4].

The algorithm is based on the notion of observation table which contains the information accumulated by the learner during the learning process. An observation table over a given alphabet $\Sigma \times \Gamma_{k}$ is a triple $\mathcal{O}=(T, U, V)$ with $U, V$ two sets of words over $\Sigma \times \Gamma_{k}$ such that $\varepsilon \in U \cap V$ and $T$ is a mapping $\left(U \cup U \cdot\left(\Sigma \times \Gamma_{k}\right)\right) \times V \rightarrow\{+,-\}$. A table is partitioned into an upper part $U$ and a lower part $U \cdot\left(\Sigma \times \Gamma_{k}\right)$. We define for each $u \in U \cup U \cdot\left(\Sigma \times \Gamma_{k}\right)$ a mapping $\operatorname{row}(u): V \rightarrow\{+,-\}$ where $\operatorname{row}(u)(v)=T(u, v)$. An observation table must satisfy the following property: for all $u, u^{\prime} \in U$ such that $u \neq u^{\prime}$ we have $\operatorname{row}(u) \neq$ $\operatorname{row}\left(u^{\prime}\right)$, i.e., there exists $v \in V$ such that $T(u, v) \neq T\left(u^{\prime}, v\right)$. This means that the rows of the upper part of the table are pairwise distinct. A table is closed if, for all $u^{\prime}$ in $U \cdot\left(\Sigma \times \Gamma_{k}\right)$, there exists $u \in U$ such that $\operatorname{row}(u)=\operatorname{row}\left(u^{\prime}\right)$. From a closed table we can construct a symbolically deterministic session automaton whose states correspond to the rows of the upper part of the table:

Definition 2. For a closed table $\mathcal{O}=(T, U, V)$ over a finite alphabet $\Sigma \times$ $\Gamma_{k}$, we define a symbolically deterministic $k$-register session automaton $A_{\mathcal{O}}=$ $\left(Q, q_{0}, F, \delta\right)$ over $\Sigma \times \Gamma_{k}$ by $Q=U, q_{0}=\varepsilon, F=\{u \in Q \mid T(u, \epsilon)=+\}$, and for all $u \in Q$ and $(a, t) \in \Sigma \times \Gamma_{k}, \delta(u,(a, t))=u^{\prime}$ if $\operatorname{row}(u(a, t))=\operatorname{row}\left(u^{\prime}\right)$. This is well defined as the table is closed.

We now describe in detail our active learning algorithm for a given session automaton $\mathcal{A}$ given in Table 1 . It is based on a loop which repeatedly constructs

\footnotetext{
${ }^{4}$ This can be checked in polynomial time over the trimmed hypothesis automaton with a fixed point computation labelling the states with the registers that should be used again before overwriting them.
} 


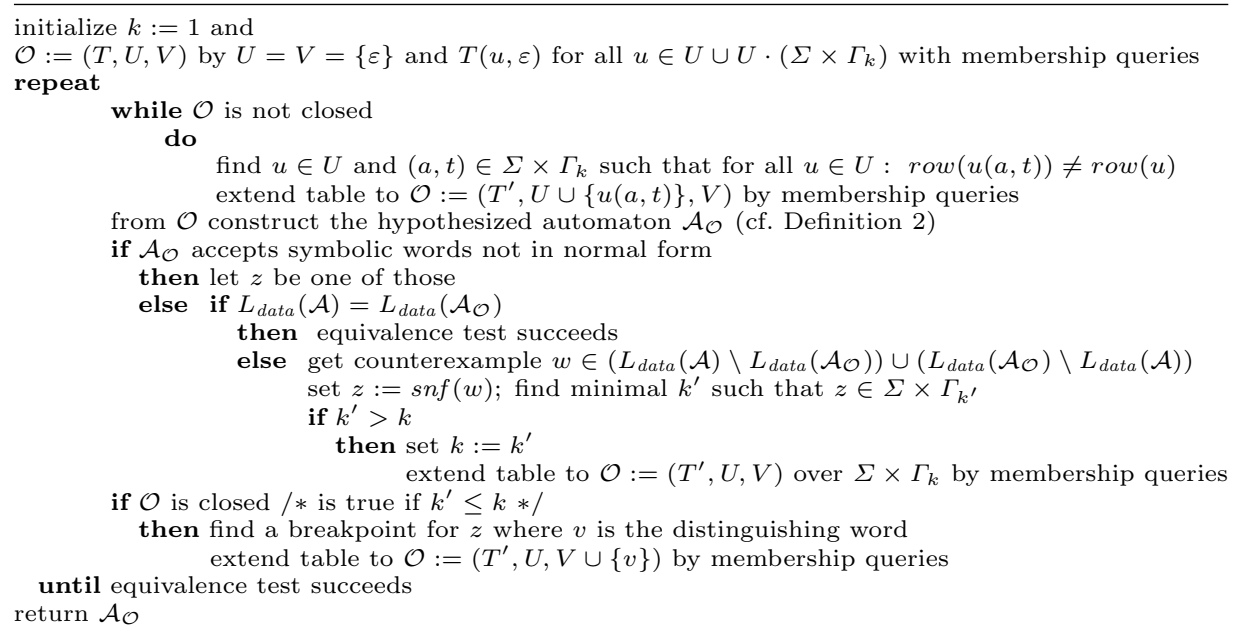

Table 1: The learning algorithm for a session automaton $\mathcal{A}$

a closed table using membership queries, builds the corresponding automaton and then asks an equivalence query. This is repeated until $\mathcal{A}$ is learned. An important part of any active learning algorithm is the treatment of counterexamples provided by the teacher as an answer to an equivalence query. Suppose that for a given $\mathcal{A}_{\mathcal{O}}$ constructed from a closed table $\mathcal{O}=(T, U, V)$ the teacher answers by a counterexample data word $w$. Let $z=\operatorname{snf}(w)$. If $z$ uses more registers than available in the current alphabet, we extend the alphabet and then the table. If the obtained table is not closed, we restart from the beginning of the loop. Otherwise - and also if $z$ does not use more registers - we use Rivest and Schapire's [22] technique to extend the table by adding a suitable $v$ to $V$ making it non-closed. The technique is based on the notion of breakpoint. As $z$ is a counterexample, $(1) z \in L_{\text {symb }}\left(\mathcal{A}_{\mathcal{O}}\right) \Longleftrightarrow z \notin L_{\text {symb }}\left(\mathcal{A}^{C}\right)$. Let $z=z_{1} \cdots z_{m}$. Then, for any $i$ with $1 \leq i \leq m+1$, let $z$ be decomposed as $z=u_{i} v_{i}$, where $u_{1}=v_{m+1}=\varepsilon, v_{1}=u_{m+1}=z$ and the length of $u_{i}$ is equal to $i-1$ (we have also $z=u_{i} z_{i} v_{i+1}$ for all $i$ such that $1 \leq i \leq m$ ). Let $s_{i}$ be the state visited by $z$ just before reading the $i$ th letter, along the computation of $z$ on $\mathcal{A}_{\mathcal{O}}: i$ is a breakpoint if $s_{i} z_{i} v_{i+1} \in L_{\text {symb }}\left(\mathcal{A}_{\mathcal{O}}\right) \Longleftrightarrow s_{i+1} v_{i+1} \notin L_{\text {symb }}\left(\mathcal{A}^{C}\right)$. Because of (1) such a break-point must exist and can be obtained with $O(\log (m))$ membership queries by a dichotomous search. The word $v_{i+1}$ is called the distinguishing word. If $V$ is extended by $v_{i+1}$ the table is not closed anymore $\left(\right.$ row $\left(s_{i}\right)$ and $\operatorname{row}\left(s_{i} z_{i}\right)$ are different). Now, the algorithm closes the table again, then asks another equivalence query and so forth until termination. At each iteration of the loop the number of rows (each of those correspond to a state in the automaton $\mathcal{A}^{C}$ ) is increased by at least one. Notice that the same counterexample might be given several times. The treatment of the counterexample only guarantees that the table will contain one more row in its upper part. We obtain the following: 
Theorem 3. Let $\mathcal{A}$ be a $k^{\prime}$-register session automaton over $\Sigma$ and D. Let $\mathcal{A}^{C}$ be the corresponding canonical $k$-register session automaton. Let $N$ be its number of states, $K$ be the size of $\Sigma \times \Gamma_{k}$ and $M$ the length of the longest counterexample returned by an equivalence query. Then, the learning algorithm for $\mathcal{A}$ terminates with at most $O\left(K N^{2}+N \log (M)\right)$ membership and $O(N)$ equivalence queries.

Proof: This follows directly from the proof of correctness and complexity of Rivest and Schapire's algorithm $[4,22]$. Notice that the equivalence query cannot return a counterexample whose normal form uses more than $k$ registers, as such a word is rejected by both $\mathcal{A}^{C}$ (by definition) and by $\mathcal{A}_{\mathcal{O}}$, (by construction).

Let us discuss the complexity of our algorithm. In terms of the canonical session automaton, the number of required membership and equivalence queries is polynomial. When we consider data deterministic session automata, the complexity is still polynomial in the number of states, but exponential in $k$ (with constant base). As usual, we have to add one exponent wrt. (data) non-deterministic automata. In [14], the number of equivalence queries is polynomial in the size of the underlying automaton. In contrast, the number of membership queries contains a factor $n^{k}$ where $n$ is the number of states and $k$ the number of registers. This may be seen as a drawback, as $n$ is typically large. Note that [14] restrict to deterministic automata, since classical register automata are not determinizable.

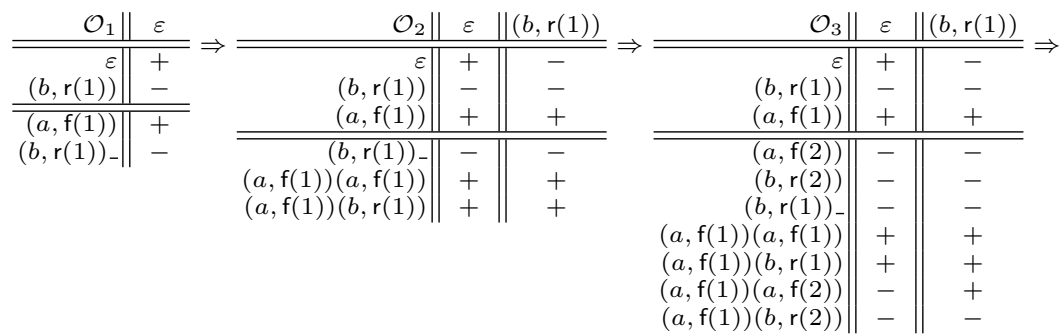

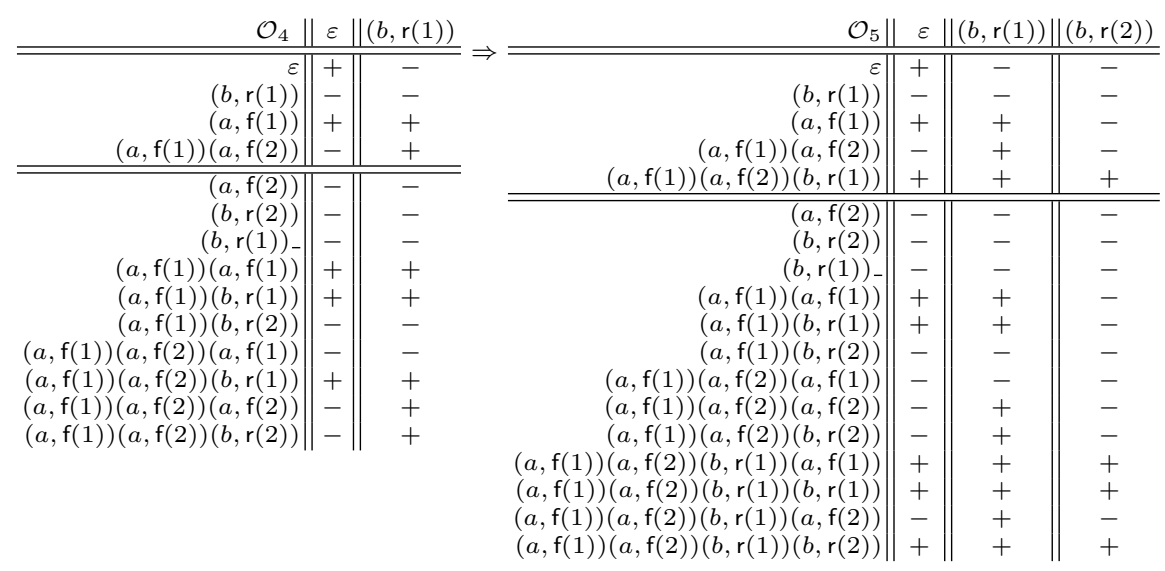

Fig. 4: The successive observation tables 


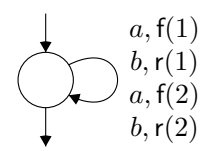

Fig. 5: The 2-register automaton to learn

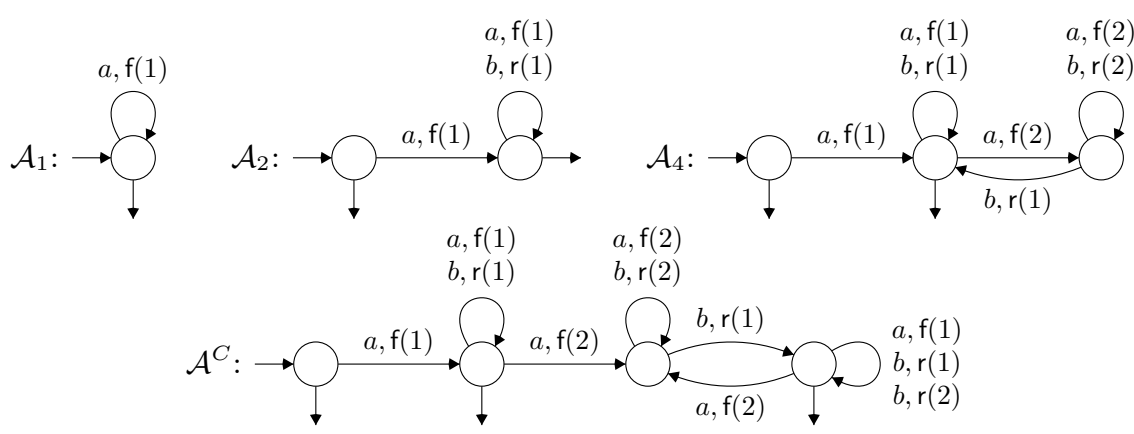

Fig. 6: The successive hypothesis automata

Example 4. We apply our learning algorithm on the data language given by the automaton $\mathcal{A}$ of Fig. 5. In Fig. 4 the successive observation tables constructed by the algorithm are given. To save space some letters whose rows contain only -'s are omitted. In Fig. 6 the successive automata constructed from the closed observation tables are given. For sake of clarity we omit the sink states. We start with the alphabet $\Sigma \times \Gamma_{1}=\{(a, \mathrm{f}(1)),(a, \mathrm{r}(1)),(b, \mathrm{f}(1)),(b, \mathrm{r}(1))\}$. We omit letters $(a, \mathrm{r}(1))$ and $(b, \mathrm{f}(1))$. Table $\mathcal{O}_{1}$ is obtained after initialization and closing by adding $(b, \mathrm{r}(1))$ to the top. We use - to indicate that all letters will lead to the same row. From $\mathcal{O}_{1}$ the first hypothesis automaton $\mathcal{A}_{1}$ is constructed. We suppose that the equivalence query gives back as counterexample the data word $(a, 3)(b, 3)$ whose normal form is $(a, \mathrm{f}(1))(b, \mathrm{r}(1))$. Here the breakpoint yields the distinguishing word $(b, \mathrm{r}(1))$. We add it to $V$. The obtained table is not closed anymore. We close it by adding $(a, \mathrm{f}(1))$ to the top and get table $\mathcal{O}_{2}$ yielding hypothesis automaton $\mathcal{A}_{2}$. Notice that $L_{\text {symb }}\left(\mathcal{A}_{2}\right)=L_{\text {symb }}\left(\mathcal{A}^{C}\right) \cap(\Sigma \times$ $\left.\Gamma_{1}\right)^{*}$. This means that the equivalence query must give back a data word whose normal form is using at least 2 registers (here $(a, 7)(a, 4)(b, 7)$ with normal form $(a, f(1))(a, f(2))(b, r(1)))$. As the word uses 2 registers, we extend the alphabet to $\Sigma \times \Gamma_{2}$ and obtain table $\mathcal{O}_{3}$. We close the table and get $\mathcal{O}_{4}$. From there we obtain the hypothesis automaton $\mathcal{A}_{4}$. After the equivalence query we get $(a, \mathrm{f}(1))(a, \mathrm{f}(2))(b, \mathrm{r}(1))(b, \mathrm{r}(2))$ as normal form of the data word counterexample $(a, 9)(a, 3)(b, 9)(b, 3)$. After adding $(b, \mathrm{r}(2))$ to $V$ and closing the table by moving $(a, \mathrm{f}(1))(a, \mathrm{f}(2))(b, \mathrm{r}(1))$ to the top we get finally the table $\mathcal{O}_{5}$ from which the canonical automaton $\mathcal{A}^{C}$ is obtained and the equivalence query succeeds. 


\section{Language Theoretical Results}

In this section, we establish some language theoretical properties of session automata, which they inherit from classical regular languages. These results demonstrate a certain robustness as required in verification tasks such as compositional verification [10] and infinite-state regular model checking [13].

Theorem 4. Data languages recognized by session automata are closed under intersection and union. They are also closed under complementation in the following sense: given a $k$-register session automaton $\mathcal{A}$, the language $\gamma\left(\left(\Sigma \times \Gamma_{k}\right)^{*}\right) \backslash$ $L_{\text {data }}(\mathcal{A})$ is recognized by a $k$-register session automaton.

Theorem 5. The inclusion problem for session automata is decidable.

We now provide a logical characterization of session automata. We consider data MSO logic (dMSO), which is an extension of classical MSO logic by the binary predicate $x \sim y$ to compare data values: a data word $w=$ $\left(a_{1}, d_{1}\right) \cdots\left(a_{n}, d_{n}\right) \in(\Sigma \times D)^{*}$ with variable interpretation $x \mapsto i$ and $y \mapsto j$ satisfies $x \sim y$ if $d_{i}=d_{j}$. For more background on dMSO, we refer the reader to the appendix and $[21,23,6]$. Note that dMSO is a very expressive logic and goes beyond virtually all automata models defined for data words $[21,6,11]$. We identify a fragment of dMSO, called session MSO logic, that is expressively equivalent to session automata. While register automata also enjoy a logical characterization [11], we are not aware of logics capturing the automata model considered in [14].

Definition 3. A session MSO (sMSO) formula is a dMSO sentence of the form $\varphi=\exists X_{1} \cdots \exists X_{m}(\alpha \wedge \forall x \forall y(x \sim y \leftrightarrow \beta))$ such that $\alpha$ and $\beta$ are classical $M S O$ formulas (not containing the predicate $\sim$ ).

Example 5. For instance, $\varphi_{1}=\forall x \forall y(x \sim y \leftrightarrow x=y)$ is an sMSO formula. Its semantics $L_{\text {data }}\left(\varphi_{1}\right)$ is the set of data words in which every data value occurs at most once. Moreover, $\varphi_{2}=\forall x \forall y(x \sim y \leftrightarrow$ true $)$ is an sMSO formula, and $L_{\text {data }}\left(\varphi_{2}\right)$ is the set of data words where all data values coincide. As a last example, let $\varphi_{3}=\exists X \forall x \forall y(x \sim y \leftrightarrow(\neg \exists z \in X(x<z \leq y \vee y<z \leq x)))$. Then, $L_{\text {data }}\left(\varphi_{3}\right)$ is the set of 1-bounded data words. Intuitively, the second-order variable $X$ represents the set of positions where a fresh data value is introduced.

Theorem 6. A data language is recognized by a session automaton iff it is definable by an sMSO formula.

In [7], it was already shown (for a more powerful model with pushdown stacks) that model checking for the full dMSO logic is decidable:

Theorem 7 ([7]). Given a session automaton $\mathcal{A}$ and a dMSO sentence $\varphi$, one can decide whether $L_{\text {data }}(\mathcal{A}) \subseteq L_{\text {data }}(\varphi)$. 


\section{Conclusion}

In this paper, we provided a complete framework for algorithmic learning of session automata, a special class of register automata to process data words. As a key ingredient, we associated with every session automaton a canonical one, which revealed close connections with classical regular languages. This also allowed us to show that session automata form a robust language class with good closure and decidability properties as well as a characterization in MSO logic. As a next step, we plan to employ our setting for various verification tasks.

Acknowledgment. We are grateful to Thomas Schwentick for suggesting the symbolic normal form of data words.

\section{References}

1. F. Aarts, F. Heidarian, H. Kuppens, P. Olsen, and F. W. Vaandrager. Automata learning through counterexample guided abstraction refinement. In $F M$, LNCS 7436, pp. 10-27. Springer, 2012.

2. D. Angluin. Learning regular sets from queries and counterexamples. Information and Computation, 75(2):87-106, 1987.

3. T. Berg, O. Grinchtein, B. Jonsson, M. Leucker, H. Raffelt, and B. Steffen. On the correspondence between conformance testing and regular inference. In FASE, LNCS 3442, pp. 175-189. Springer, 2005.

4. T. Berg and H. Raffelt. Model checking. In Model-based Testing of Reactive Systems, LNCS 3472 of LNCS. Springer, 2005.

5. H. Björklund and Th. Schwentick. On notions of regularity for data languages. Theoretical Computer Science, 411(4-5):702-715, 2010.

6. M. Bojanczyk, C. David, A. Muscholl, T. Schwentick, and L. Segoufin. Twovariable logic on data words. ACM Trans. Comput. Log., 12(4):27, 2011.

7. B. Bollig, A. Cyriac, P. Gastin, and K. Narayan Kumar. Model checking languages of data words. In FoSSaCS, LNCS 7213, pp. 391-405. Springer, 2012.

8. B. Bollig, J.-P. Katoen, C. Kern, M. Leucker, D. Neider, and D. Piegdon. libalf: the automata learning framework. In $C A V$, LNCS 6174, pp. 360-364. Springer, 2010.

9. S. Cassel, F. Howar, B. Jonsson, M. Merten, and B. Steffen. A succinct canonical register automaton model. In ATVA, LNCS 6996, pp. 366-380. Springer, 2011.

10. J. M. Cobleigh, D. Giannakopoulou, and C. S. Pasareanu. Learning assumptions for compositional verification. In TACAS, LNCS 2619, pp. 331-346. Springer, 2003.

11. T. Colcombet, C. Ley, and G. Puppis. On the use of guards for logics with data. In Proceedings of MFCS, LNCS 6907, pp. 243-255. Springer, 2011.

12. D. Giannakopoulou and J. Magee. Fluent model checking for event-based systems. In ESEC / SIGSOFT FSE, pp. 257-266. ACM, 2003.

13. P. Habermehl and T. Vojnar. Regular Model Checking Using Inference of Regular Languages. In INFINITY'04, ENTCS 138, pp. 21-36. Elsevier, 2005.

14. F. Howar, B. Steffen, B. Jonsson, and S. Cassel. Inferring canonical register automata. In VMCAI, LNCS 7148, pp. 251-266. Springer, 2012.

15. B. Jonsson. Learning of automata models extended with data. In SFM, LNCS 6659, pp. 327-349. Springer, 2011.

16. M. Kaminski and N. Francez. Finite-memory automata. Theoretical Computer Science, 134(2):329-363, 1994. 
17. K. O. Kürtz, R. Küsters, and T. Wilke. Selecting theories and nonce generation for recursive protocols. In FMSE, pp. 61-70. ACM, 2007.

18. M. Leucker. Learning meets verification. LNCS 4709, pp. 127-151. Springer, 2007.

19. T. Margaria, H. Raffelt, B. Steffen, and M. Leucker. The LearnLib in FMICS-jETI. In ICECCS, pp. 340-352. IEEE Computer Society Press, 2007.

20. R. Milner, J. Parrow, and D. Walker. A calculus of mobile processes, Parts I and II. Information and Computation, 100:1-77, Sept. 1992.

21. F. Neven, Th. Schwentick, and V. Vianu. Finite state machines for strings over infinite alphabets. ACM Transactions on Computational Logic, 5(3):403-435, 2004.

22. R. Rivest and R. Schapire. Inference of finite automata using homing sequences. Information and Computation, 103:299-347, 1993.

23. L. Segoufin. Automata and logics for words and trees over an infinite alphabet. In Z. Ésik, editor, CSL, LNCS 4207, pp. 41-57. Springer, 2006.

24. N. Tzevelekos. Fresh-register automata. In POPL, pp. 295-306. ACM, 2011. 


\section{Appendix}

\section{A Proofs for Section 2}

This section is devoted to the construction of the canonical session automata as shortly described in Section 2.

We first start with some global remarks about the definition of the symbolic normal forms. Let $w \in(\Sigma \times D)^{*}$. Then, $w$ is $k$-bounded iff $\operatorname{snf}(w) \in\left(\Sigma \times \Gamma_{k}\right)^{*}$. Moreover, for all data languages $L$, we have $L=\gamma(\operatorname{snf}(L))$. Note that $w \approx w^{\prime}$ implies $\operatorname{snf}(w)=\operatorname{snf}\left(w^{\prime}\right)$, which validates our choice of denomination for snf. Indeed, two equivalent data words $w$ and $w^{\prime}$ have the same set of sessions (in particular, Fresh $\left.(w)=\operatorname{Fresh}\left(w^{\prime}\right)\right)$ and the same $\Sigma$-labels. Thus, the mappings $\tau$ used to define $\operatorname{snf}(w)$ and $\operatorname{snf}\left(w^{\prime}\right)$ are the same and $\operatorname{snf}(w)=\operatorname{snf}\left(w^{\prime}\right)$.

As an illustration of the definition, let us consider the language $\mathrm{NF}_{k} \stackrel{\text { def }}{=}$ $\operatorname{snf}\left(\gamma\left(\left(\Sigma \times \Gamma_{k}\right)^{*}\right)\right)$ consisting of the symbolic normal forms of all $k$-bounded data words. We explain how to construct a symbolically deterministic $k$-register session automaton $\mathcal{A}$ such that $L_{\text {symb }}(\mathcal{A})=\mathrm{NF}_{k}$. Its state space is $Q=(\{0\} \cup$ $[k]) \times \subset[k]$, consisting of (i) the greatest register already initialized (indeed we will only use a register $r$ if every register $r^{\prime}<r$ has already been used), (ii) a subset $P$ of registers that we promise to reuse again before resetting their value. The initial state of $\mathcal{A}$ is $(0, \emptyset)$, the set of accepting states is $(\{0\} \cup[k]) \times\{\emptyset\}$. We now describe the set of transitions. For every $a \in \Sigma, i \in\{0\} \cup[k], P \subseteq[k]$, and $r \in[k]:$

$$
\begin{aligned}
& \delta((i, P),(a, \mathrm{r}(r)))= \begin{cases}\{(i, P \backslash\{r\})\} & \text { if } r \leq i \\
\emptyset & \text { otherwise }\end{cases} \\
& \delta((i, P),(a, \mathrm{f}(r)))= \begin{cases}\{(\max (i, r), P \cup[r-1])\} & \text { if } r-1 \leq i \wedge r \notin P \\
\emptyset & \text { otherwise }\end{cases}
\end{aligned}
$$

Fig. 7 depicts the 2-register session automaton for $\mathrm{NF}_{2}$ (omitting $\Sigma$ ).

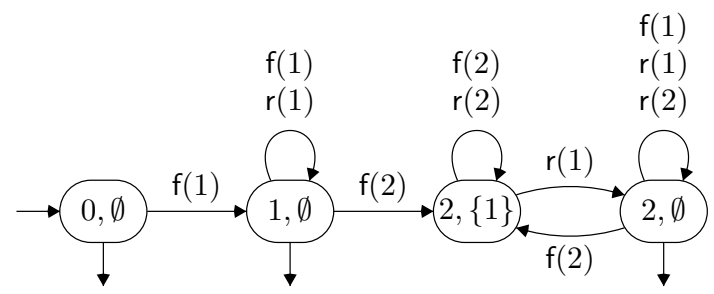

Fig. 7: A 2-register session automaton recognizing $\mathrm{NF}_{2}$

This example permits to prove Theorem 1:

Theorem 1. Session automata are strictly more expressive than data deterministic session automata. 
Proof: We show that the data language $L=\gamma\left(\mathrm{NF}_{2}\right)$ cannot be recognized by a data deterministic session automaton. Indeed, suppose that such an automaton exists, with $k$ registers. Then, consider the word $w=(a, 1)(a, 2)(a, 3) \cdots(a, k+$ $1) \in L$, where every data value is fresh. By data determinism, there is a unique run accepting $w$. Along this run, let $i<j$ be two positions such that their two fresh data values have been stored in the same register $r$ (such a pair must exist since the automaton has only $k$ registers). Without loss of generality, we can consider the greatest position $j$ verifying this condition, and then the greatest position $i$ associated with $j$. This means that register $r$ is used for the last time when reading $j$, and has not been used in-between positions $i$ and $j$. Now, the word $(a, 1)(a, 2)(a, 3) \cdots(a, k+1)(a, i) \in L$ must be recognized by the automaton, but cannot since data value $i$ appearing on the last position is not fresh anymore, and yet not stored in one of the registers (since register $r$ was reused at $j$ ).

The example $\mathrm{NF}_{k}$ also shows that regularity of the symbolic language $(\Sigma \times$ $\left.\Gamma_{k}\right)^{*}$ is preserved under $\operatorname{snf}(\gamma()$.$) . We now prove that this is the case for every$ regular language over $\Sigma \times \Gamma_{k}$. The proof will actually use the special case of $\mathrm{NF}_{k}$.

Lemma 1. Let $L$ be a regular language over $\Sigma \times \Gamma_{k}$. Then, $\operatorname{snf}(\gamma(L))$ is a regular language over $\Sigma \times \Gamma_{k}$.

Proof: Let $L \subseteq\left(\Sigma \times \Gamma_{k}\right)^{*}$ be regular. Consider the language $\tilde{L}=\{u \in \mathrm{WF} \cap(\Sigma \times$ $\left.\Gamma_{k}\right)^{*} \mid$ there is $u^{\prime} \in L$ such that $\left.\gamma(u)=\gamma\left(u^{\prime}\right)\right\}$, i.e., the set of well formed symbolic words having the same concretizations as some word from $L$. We can easily show that $\operatorname{snf}(\gamma(L))=\mathrm{NF}_{k} \cap \tilde{L}$. Indeed, if $u \in \operatorname{snf}(\gamma(L))$, then there are $u^{\prime} \in L$ and $w \in \gamma\left(u^{\prime}\right)$ such that $u=\operatorname{snf}(w)$. Necessarily, $w$ is $k$-bounded. Hence, $u \in \mathrm{NF}_{k}$. Moreover, we have $[w]_{\approx}=\gamma\left(u^{\prime}\right)$ (by Remark 1) and $w \in \gamma(\operatorname{snf}(w))=\gamma(u)$ implying also $[w]_{\approx}=\gamma(u)$. Finally, we obtain $\gamma(u)=\gamma\left(u^{\prime}\right)$. Reciprocally, if $u \in \mathrm{NF}_{k} \cap \tilde{L}$, then there is $u^{\prime} \in L$ such that $\gamma(u)=\gamma\left(u^{\prime}\right)$. Hence, starting from any word $w$ in $\gamma(u)$, we have $u=\operatorname{snf}(w)$ (by uniqueness of the symbolic normal form) and $w \in \gamma\left(u^{\prime}\right) \subseteq \gamma(L)$, so that $u \in \operatorname{snf}(\gamma(L))$.

We know from previous discussions that $\mathrm{NF}_{k}$ is regular. It remains to be shown that $\tilde{L}$ is regular. To do so, let $\mathcal{A}=\left(Q, q_{0}, F, \delta\right)$ be a $k$-register session automaton such that $L_{\text {symb }}(\mathcal{A})=L$. We construct a $k$-register session automaton $\tilde{\mathcal{A}}=\left(Q \times \operatorname{Inj}(k),\left(q_{0}, \emptyset\right), F \times \operatorname{Inj}(k), \tilde{\delta}\right)$ recognizing the symbolic language $\tilde{L}$. Hereby, $\operatorname{Inj}(k)$ is the set of partial injective mappings from $[k]$ to $[k]$, and $\emptyset \in$ $\operatorname{Inj}(k)$ denotes the mapping with empty domain. These partial mappings are used to remember the correspondence between old register indices and new ones, so they may be understood as a set of constraints. For example, the mapping $(2 \mapsto 1,1 \mapsto 3)$ stands for "old register 2 henceforth refers to 1 , and old register 1 henceforth refers to 3". Any subset of these constraints forms always a valid partial injective mapping. In the following, such a subset is called a sub-mapping. For example, $\pi=(1 \mapsto 3)$ is a sub-mapping of the previous one; it can then be extended with the new constraint $2 \mapsto 2$, which we denote $\pi[2 \mapsto 2]$. We describe 


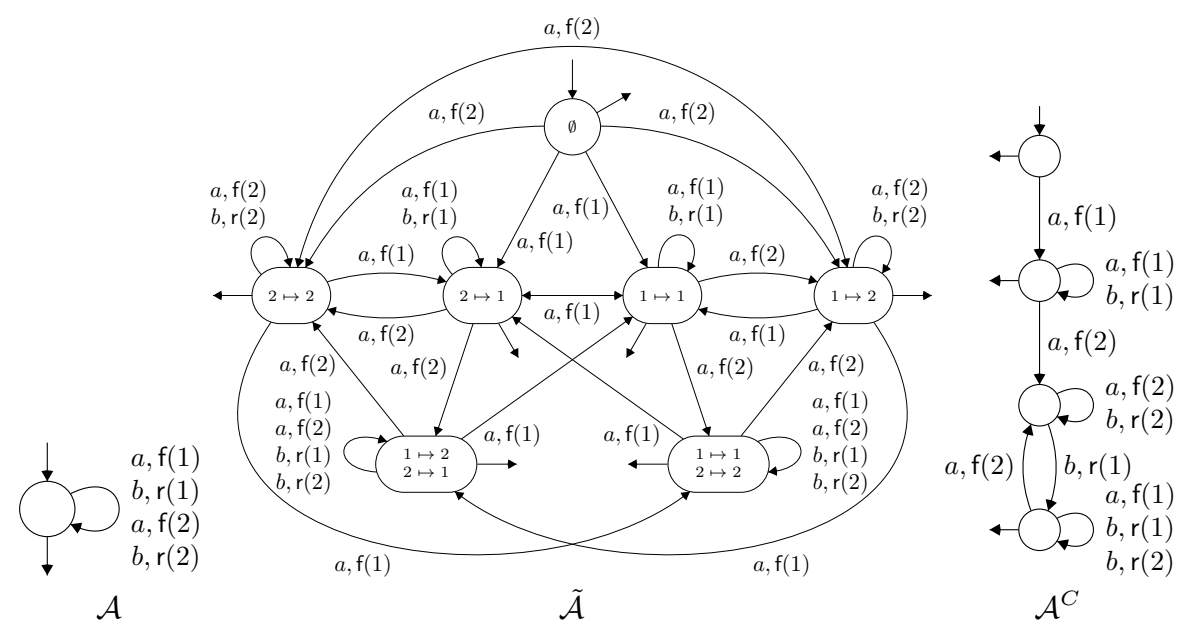

Fig. 8: A 2-register automaton, automaton $\tilde{\mathcal{A}}$, and the canonical automaton $\mathcal{A}^{C}$

now the transition relation of $\tilde{\mathcal{A}}$ :

$$
\begin{aligned}
\tilde{\delta}((q, \pi),(a, \mathrm{r}(\pi(r)))) & =\left\{\left(q^{\prime}, \pi\right) \mid q^{\prime} \in \delta(q,(a, \mathrm{r}(r)))\right\} \\
\tilde{\delta}\left((q, \pi),\left(a, \mathrm{f}\left(r^{\prime}\right)\right)\right) & =\left\{\left(q^{\prime}, \pi^{\prime}\right) \mid q^{\prime} \in \delta(q,(a, \mathrm{f}(r))) \wedge \pi^{\prime}=\pi^{\prime \prime}\left[r \mapsto r^{\prime}\right]\right. \\
& \text { with } \left.\pi^{\prime \prime} \text { maximal sub-mapping of } \pi \text { s.t. } \pi^{\prime \prime}\left[r \mapsto r^{\prime}\right] \text { injective }\right\}
\end{aligned}
$$

We simulate $r$-transitions simply using the current mapping $\pi$. For $f$-transitions, we update $\pi$, recording the new permutation of the registers. One can indeed show that $L_{\text {symb }}(\tilde{\mathcal{A}})=\tilde{L}$.

Building the product of the automaton recognizing $\mathrm{NF}_{k}$ and the automaton $\tilde{\mathcal{A}}$, we obtain a $k$-register session automaton recognizing $\operatorname{snf}(\gamma(L))$. Its number of states is bounded above by $O\left(|Q| \times k ! \times(k+1) \times 2^{k}\right.$ ) (as the number of partial injective mappings in $\operatorname{Inj}(k)$ is bounded above by $O(k !))$.

From the constructions of the automata for $\mathrm{NF}_{k}$ and $\tilde{L}$ (proof of Lemma 1), we can infer upper bounds on the size of the canonical session automaton.

Theorem 2. Let $\mathcal{A}=\left(Q, q_{0}, F, \delta\right)$ be a k-register session automaton. Then, $\mathcal{A}^{C}$ has at most $2^{O\left(|Q| \times(k+1) ! \times 2^{k}\right)}$ states. If $\mathcal{A}$ is data deterministic, then $\mathcal{A}^{C}$ has at most $O\left(|Q| \times(k+1) ! \times 2^{k}\right)$ states. Finally, $\mathcal{A}^{C}$ uses at most $k$ registers.

Example 6. Examples of $\mathcal{A}$ and $\tilde{\mathcal{A}}$, as defined in the previous proof, are given in Fig. 8. The figure also depicts the canonical automaton $\mathcal{A}^{C}$ associated with $\mathcal{A}$, obtained by determinizing and minimizing the product of both $\tilde{\mathcal{A}}$ and the deterministic automaton recognizing $\mathrm{NF}_{2}$ (as given in Fig. 7). Note that $\mathcal{A}^{C}$ is symbolically deterministic and minimal. 


\section{B Proofs for Section 4}

\section{B.1 Closure Properties and Decision Problems}

Theorem 4. Data languages recognized by session automata are closed under intersection and union. They are also closed under complementation in the following sense: given a $k$-register session automaton $\mathcal{A}$, the language $\gamma\left(\left(\Sigma \times \Gamma_{k}\right)^{*}\right) \backslash$ $L_{\text {data }}(\mathcal{A})$ is recognized by a $k$-register session automaton.

Proof: Let $\mathcal{A}$ be a $k$-register session automaton, and $\mathcal{B}$ a $k^{\prime}$-register session automaton. Using a classical product construction for $\mathcal{A}^{C}$ and $\mathcal{B}^{C}$, we obtain a $\min \left(k, k^{\prime}\right)$-register session automaton recognizing the data language $L_{\text {data }}(\mathcal{A}) \cap$ $L_{\text {data }}(\mathcal{B})$. The language $L_{\text {data }}(\mathcal{A}) \cup L_{\text {data }}(\mathcal{B})$ is recognized by the $\max \left(k, k^{\prime}\right)$ register session automaton that we obtain as the "disjoint union" of $\mathcal{A}$ and $\mathcal{B}$, branching on the first transition in one of these two automata.

Finally, let us consider a symbolically deterministic $k$-register session automaton $\mathcal{A}$. Without loss of generality, by adding a sink state, we can suppose that $\mathcal{A}$ is complete. Then, every well formed symbolic word over $\Sigma \times \Gamma_{k}$ has exactly one run in $\mathcal{A}$. The automaton $\mathcal{A}^{\prime}$ constructed from $\mathcal{A}$ by taking as accepting states the non-accepting states of $\mathcal{A}$ verifies that $L_{\text {symb }}\left(\mathcal{A}^{\prime}\right)=\left(\Sigma \times \Gamma_{k}\right)^{*} \backslash L_{\text {symb }}(\mathcal{A})$ so that $L_{\text {data }}\left(\mathcal{A}^{\prime}\right)=\gamma\left(\left(\Sigma \times \Gamma_{k}\right)^{*}\right) \backslash L_{\text {data }}(\mathcal{A})$.

Theorem 5. The inclusion problem for session automata is decidable.

Proof: Considering two session automata $\mathcal{A}$ and $\mathcal{B}$, we can decide inclusion $L_{\text {data }}(\mathcal{A}) \subseteq L_{\text {data }}(\mathcal{B})$ by considering the canonical automata $\mathcal{A}^{C}$ and $\mathcal{B}^{C}$. Indeed, $L_{\text {data }}(\mathcal{A}) \subseteq L_{\text {data }}(\mathcal{B}) \Longleftrightarrow \operatorname{snf}\left(L_{\text {data }}(\mathcal{A})\right) \subseteq \operatorname{snf}\left(L_{\text {data }}(\mathcal{B})\right) \Longleftrightarrow L_{\text {symb }}\left(\mathcal{A}^{C}\right) \subseteq$ $L_{\text {symb }}\left(\mathcal{B}^{C}\right)$. Thus, it is sufficient to check inclusion for $\mathcal{A}^{C}$ and $\mathcal{B}^{C}$.

\section{B.2 Logical Characterization}

We fix infinite supplies of first-order variables $x, y, \ldots$, which are interpreted as word positions, and second-order variables $X, Y, \ldots$, which are taken as sets of positions. Atomic dMSO formulas are $x=y$, label $(x)=a$ (with $a \in \Sigma$ ), $y=$ $x+1$, and $x \in X$. They allow us to reason about word positions in the expected manner: given a data word $w=\left(a_{1}, d_{1}\right) \cdots\left(a_{n}, d_{n}\right) \in(\Sigma \times D)^{*}$, positions $i, j \in$ $[n]$, and a set $I \subseteq[n]$, we have $w, i, j=x=y$ (to be read as " $w$ satisfies $x=y$ when $x$ is interpreted as $i$ and $y$ as $j ")$ if $i=j ; w, i \models \operatorname{label}(x)=a$ if $a_{i}=a$; $w, i, j \models y=x+1$ if $j=i+1$; and $w, i, I \models x \in X$ if $i \in I$. To reason about data, there is another atomic formula, $x \sim y$, which compares data values at $x$ and $y$. More precisely, $w, i, j \models x \sim y$ if $d_{i}=d_{j}$. Finally, dMSO logic provides negation and disjunction, as well as existential quantifiers $\exists x \varphi$ and $\exists X \varphi$ (with $\varphi$ a dMSO formula), interpreted as usual. In addition, we use abbreviations such as true, $x \leq y, \forall x \varphi, \varphi \wedge \psi, \varphi \rightarrow \psi$, etc. A sentence is a formula without free variables. For a dMSO sentence $\varphi$, we set $L_{\text {data }}(\varphi) \stackrel{\text { def }}{=}\left\{w \in(\Sigma \times D)^{*}|w|=\varphi\right\}$. We denote by MSO the fragment of dMSO not making use of $\sim$. An MSO formula can also be interpreted over words from $\Sigma^{*}$, without data. For an MSO sentence $\varphi$, we let $L_{\text {symb }}(\varphi) \subseteq \Sigma^{*}$ denote the set of words $w \in \Sigma^{*}$ such that $w \models \varphi$. 
Theorem 6. A data language is recognized by a session automaton iff it is definable by an sMSO formula.

The construction of an sMSO formula $\exists X_{1} \cdots \exists X_{m}(\alpha \wedge \forall x \forall y(x \sim y \leftrightarrow \beta))$ from a session automaton $\mathcal{A}$ was implicitly shown in [7] (with a different goal, though). The idea is that the existential second-order variables $X_{1}, \ldots, X_{m}$ are used to guess an assignment of transitions to positions. In $\alpha$, it is verified that the assignment corresponds to a run of $\mathcal{A}$. Moreover, $\beta$ checks if data equality corresponds to the data flow as enforced by the transition labels from $\Gamma_{k}$.

Let us turn to the converse direction, from logic to automata. Fix an sMSO formula $\varphi=\exists X_{1} \cdots \exists X_{m}(\alpha \wedge \forall x \forall y(x \sim y \leftrightarrow \beta))$. Then, $\beta$ is a formula whose free variables are among $x, y, X_{1}, \ldots, X_{m}$. As, moreover, $\beta$ is an MSO formula, which does not contain $\sim$, we can consider words over the finite alphabet $\Sigma \times\{0,1\}^{m+2}$ as models of $\beta$. The idea is to interpret a position carrying letter $\left(a, 1, b, b_{1}, \ldots, b_{m}\right)$ as $x$, and a position labeled $\left(a, b, 1, b_{1}, \ldots, b_{m}\right)$ as $y$, while membership in $X_{i}$ is indicated by $b_{i}$. Words where $x$ and $y$ are not uniquely determined, are disregarded. We can represent such models as tuples $\left(w, i, j, I_{1}, \ldots, I_{m}\right)$ where $w \in \Sigma^{*}, i$ denotes the position of the 1-entry in the unique first component, and $j$ denotes the position of the 1-entry in the second component. As $L_{\text {symb }}(\beta) \subseteq\left(\Sigma \times\{0,1\}^{m+2}\right)^{*}$ is MSO definable, it is, by Büchi's theorem, recognized by some minimal deterministic finite automaton $\mathcal{A}_{\beta}$. Suppose that $\mathcal{A}_{\beta}$ has $k_{\beta} \geq 1$ states. We fix this $k_{\beta}$ for the remainder of the section.

Proposition 1. The data language $L_{\text {data }}(\varphi)$ is $k_{\beta}$-bounded.

Proof: Let $w=\left(a_{1}, d_{1}\right) \cdots\left(a_{n}, d_{n}\right) \in L_{\text {data }}(\varphi)$. There exists a tuple $\bar{I}=$ $\left(I_{1}, \ldots, I_{m}\right)$ of subsets of $[n]$ such that, for all $i, j \in[n]$,

$$
d_{i}=d_{j} \Longleftrightarrow\left(a_{1} \cdots a_{n}, i, j, \bar{I}\right) \in L_{\text {symb }}(\beta) .
$$

Suppose, towards a contradiction, that $w$ is not $k_{\beta}$-bounded. Then, there are $k>k_{\beta}$ and a position $i \in[n]$ such that $i$ is contained in distinct sessions $S_{1}, \ldots, S_{k}$. For $l \in\{1, \ldots, k\}$, let $i_{l}=\min \left(S_{l}\right)$ and $j_{l}=\max \left(S_{l}\right)$. Note that the $i_{l}$ are pairwise distinct, and so are the $j_{l}$. For every $l \in\{1, \ldots, k\}$, we have $w_{l}=\left(a_{1} \cdots a_{n}, i_{l}, j_{l}, \bar{I}\right) \in L_{\text {symb }}(\beta)$. Thus, for every such word $w_{l}$, there is a unique accepting run of $\mathcal{A}_{\beta}$, say, being in state $q_{l}$ after executing position $i$. As $\mathcal{A}_{\beta}$ has only $k_{\beta}$ states, there are $l \neq l^{\prime}$ such that $q_{l}=q_{l^{\prime}}$. Thus, there is an accepting run of $\mathcal{A}_{\beta}$ either on a word where one of the first-order components is not unique, which is a contradiction, or on $\left(a_{1} \cdots a_{n}, i_{l}, j_{l^{\prime}}, \bar{I}\right)$. The latter contradicts $(*)$, since $S_{l}$ and $S_{l^{\prime}}$ are distinct sessions.

Next, we construct a (data-free) MSO sentence $\varphi^{\prime}$ over the alphabet $\Sigma \times$ $\Gamma_{k_{\beta}}\left(\right.$ recall that $\left.\Gamma_{k_{\beta}}=\{\mathrm{f}, \mathrm{r}\} \times\left[k_{\beta}\right]\right)$ such that $\gamma\left(L_{\text {symb }}\left(\varphi^{\prime}\right)\right)=L_{\text {data }}(\varphi)$. We will need some additional macro MSO formulas over $\Sigma \times \Gamma_{k_{\beta}} \times\{0,1\}^{m+2}$. For a first-order variable $x$ and $r \in\left\{1, \ldots, k_{\beta}\right\}$, we let $\operatorname{fresh}_{r}(x)$ stand for $\bigvee_{a, \bar{b}}$ label $(x)=(a, \mathrm{f}(r), \bar{b})$. Moreover, we let $\operatorname{reg}(x)=r$ be an abbreviation for $\operatorname{fresh}_{r}(x) \vee \bigvee_{a, \bar{b}} \operatorname{label}(x)=(a, \mathrm{r}(r), \bar{b})$. Now, we define

$$
\varphi^{\prime}=\exists X_{1} \cdots \exists X_{m}\left(\alpha^{\prime} \wedge \forall x \forall y\left(\operatorname{equal}(x, y) \leftrightarrow \beta^{\prime}\right)\right)
$$


where

$$
\operatorname{equal}(x, y)=\bigvee_{r \in\left[k_{\beta}\right]}\left(\begin{array}{c}
\operatorname{reg}(x)=r \wedge \operatorname{reg}(y)=r \\
\wedge \neg \exists z\left(\operatorname{fresh}_{r}(z) \wedge \min (x, y)<z \leq \max (x, y)\right)
\end{array}\right)
$$

and $\alpha^{\prime}$ and $\beta^{\prime}$ are obtained from $\alpha$ and $\beta$, respectively, by replacing label $(x)=a$ with $\bigvee_{t, \bar{b}} \operatorname{label}(x)=(a, t, \bar{b})$, with $\bar{b}$ ranging over $\{0,1\}^{m}$ in $\alpha^{\prime}$ and over $\{0,1\}^{m+2}$ in $\beta^{\prime}$.

Proposition 2. We have $\gamma\left(L_{\text {symb }}\left(\varphi^{\prime}\right)\right)=L_{\text {data }}(\varphi)$.

Proof: Let $u=\left(a_{1}, t_{1}\right) \cdots\left(a_{n}, t_{n}\right) \in L_{\text {symb }}\left(\varphi^{\prime}\right)$ be a well formed word, and let $w=\left(a_{1}, d_{1}\right) \cdots\left(a_{n}, d_{n}\right) \in \gamma(u)$. We will show $w \models \varphi$. There is a tuple $\bar{I}=\left(I_{1}, \ldots, I_{m}\right)$ of subsets of $[n]$ such that $u, \bar{I} \models \alpha^{\prime} \wedge \forall x \forall y$ (equal $\left.(x, y) \leftrightarrow \beta^{\prime}\right)$. This already implies $w, \bar{I}=\alpha$. Now take two positions $i, j \in[n]$. By the definition of equal $(x, y)$ and $\gamma$, we have $d_{i}=d_{j}$ iff $u, i, j, \bar{I} \models \operatorname{equal}(x, y)$ iff $u, i, j, \bar{I} \models \beta^{\prime}$ iff $w, i, j, \bar{I}=\beta$. We conclude $w=\varphi=\exists X_{1} \cdots \exists X_{m}(\alpha \wedge \forall x \forall y(x \sim y \leftrightarrow \beta))$.

For the converse direction, suppose $w=\left(a_{1}, d_{1}\right) \cdots\left(a_{n}, d_{n}\right) \in L_{\text {data }}(\varphi)$. There is a tuple $\bar{I}=\left(I_{1}, \ldots, I_{m}\right)$ of subsets of $[n]$ such that $w, \bar{I}=\alpha \wedge$ $\forall x \forall y(x \sim y \leftrightarrow \beta)$. Therefore, it already holds $u, \bar{I} \models \alpha^{\prime}$. By Proposition $1, w$ is $k_{\beta}$-bounded. Let $u=\operatorname{snf}(w)=\left(a_{1}, t_{1}\right) \cdots\left(a_{n}, t_{n}\right)$. We have $w \in \gamma(u)$ which implies $u \in\left(\Sigma \times \Gamma_{k_{\beta}}\right)^{*}$. It remains to be shown that $u, \bar{I}=\forall x \forall y$ (equal $\left.(x, y) \leftrightarrow \beta^{\prime}\right)$. Take any two positions $i, j \in[n]$. We have $u, i, j, \bar{I}=\operatorname{equal}(x, y)$ iff $d_{i}=d_{j}$. The latter is the case iff $w, i, j, \bar{I} \models \beta$ (since $w, \bar{I} \models \forall x \forall y(x \sim y \leftrightarrow \beta)$ ), which holds iff $u, i, j, \bar{I}=\beta^{\prime}$. Thus, $u \models \varphi^{\prime}$.

By Büchi's theorem, we can translate $\varphi^{\prime}$ into a finite automaton $\mathcal{A}$ recognizing the models of $\varphi^{\prime}$, which form a language over $\Sigma \times \Gamma_{k_{\beta}}$. By Proposition 2, interpreting $\mathcal{A}$ as a session automaton, we obtain $L_{\text {data }}(\mathcal{A})=L_{\text {data }}(\varphi)$. 